\title{
Le vécu des dissections au laboratoire d'anatomie. \\ Conséquences psychologiques et actions pédagogiques
}

\section{The real-life experience of dissections in a laboratory of anatomy. Psychological consequences and pedagogical measures}

\author{
Angélique BONNAUD-ANTIGNAC ${ }^{1,2}$, Olivier ARMSTRONG ${ }^{2,3}$, Antoine HAMEL ${ }^{3}$, \\ Jean-Michel ROGEZ ${ }^{3}$, Dominique GUILLOTON ${ }^{4}$, Yves MAUGARS ${ }^{2}$ \\ et Jacques-Henri BARRIER ${ }^{2}$
}

1 Département de sciences humaines et sociales, Faculté de médecine de l'université de Nantes, 1 rue Gaston Veil, 44093 Nantes Cedex, France.

2 Département de formation médicale continue et développement pédagogique, Faculté de médecine de l'université de Nantes, 1 rue Gaston Veil, 44093 Nantes Cedex, France.

3 Laboratoire d'anatomie, Faculté de médecine de Nantes, 1 rue Gaston Veil, 44093 Nantes Cedex, France.

4 École d'infirmières de bloc opératoire, 50 rue de Saint Sébastien, CHU de Nantes, 44093 Nantes, France.

Manuscrit reçu le 28 septembre 2007; commentaires éditoriaux formulés aux auteurs le 5 août 2008 ; accepté pour publication le 5 septembre 2008

\section{Mots clés : \\ Cadavre ; \\ laboratoire d'anatomie ; dissections, étudiant(e)s en médecine ; infirmier(e)s de bloc opératoire}

\section{Keywords:}

Cadaver;

laboratory of anatomy; dissections; medical students; operating room nurse students
Résumé - Contexte : Les travaux pratiques de dissections sont toujours l'objet de réactions particulières avant, pendant ou après la rencontre avec les cadavres. But : Mieux comprendre dans une perspective comparative ce qui se passe chez les jeunes étudiant(e)s de seconde année de médecine qui sont souvent vierges de toute expérience de la mort et chez des étudiant(e)s infirmier(e)s de bloc opératoire qui en plus de leur expérience personnelle ont souvent une expérience professionnelle. Méthode : Deux focus groupes, l'un avec 12 étudiant(e)s de seconde année de premier cycle des études médicales (PCEM2) et l'autre avec 12 étudiant(e)s infirmier(e)s de bloc opératoire (IBODE) explorant les mêmes dimensions. Résultats : Les IBODE expriment plus facilement leurs appréhensions que les étudiant(e)s en médecine. Tous se protègent du caractère déstabilisant de la dissection par des réactions spécifiques. Conclusion : Une séance d'information pratique et technique suivie d'une séance d'expression du ressenti est souhaitée par les deux populations.

Abstract - Context: Dissections in laboratory of anatomy are always the object of specific reactions before, during or after exposure with a cadaver. Aim: To better understand, in a comparative manner, what arises in young 2 nd year medical students, who are often virgin of any death experience, with nurse students specialized in the operating room, who have in addition to their personal experience, a professional experience. Method: Two focus groups exploring the same dimensions, one including 12 2nd year medical students and the other comprising 12 operating room nurse students. Results: The nurse students express more 
easily their apprehension than 2nd year medical students. All students protect themselves from the destabilizing nature of dissections by specific reactions. Conclusion: Practical and technical information sessions before dissections followed by a debriefing session where students can express their resentment, is desirable for both groups of students.

Les réactions face au cadavre et à la mort sont des thématiques très peu souvent abordées au cours des enseignements formels durant les études de médecine et la première expérience de confrontation des étudiants à la mort au cours de leur cursus se passe souvent à travers le cadavre en tant que sujet anatomique.

\section{Contexte et problématique pédagogique}

Après une première année de premier cycle d'études médicales (PCEM1) caractérisée par un enseignement exclusivement théorique, les étudiant(e)s français de médecine abordent la seconde année du premier cycle des études médicales (PCEM2) avec une attente qui est celle de la rencontre avec la pratique médicale et les patients. Et pourtant ce ne sont pas les patients qu'ils vont rencontrer en premier. En effet, parmi les enseignements fondamentaux l'anatomie se fait en cours magistral mais également à l'occasion de travaux pratiques de dissections sur du matériel humain. Ces travaux pratiques (TP) de dissections obligatoires sont souvent vécus avec beaucoup d'appréhension préalable. A posteriori, des réactions sont soit exprimées, soit étouffées. Les étudiant(e)s qui se destinent à la profession d'infirmier(e)s de bloc opératoire diplômés d'état (IBODE) sont, pour leur part, déjà des professionnels de santé qui vont se spécialiser après un parcours professionnel en tant qu'infirmier(e)s dans différents services de médecine. Ce sont donc des adultes qui ont réfléchi à une nouvelle orientation professionnelle dont l'apprentissage est centré sur l'anatomie de la personne à partir de cours théoriques et pratiques. La question que nous nous sommes posée est celle du vécu et des réactions de ces deux populations aux TP de dissections compte tenu d'un parcours différent.

\section{Objectifs}

L'expérience de la dissection en deuxième année des études médicales (PCEM2) est vécue plus ou moins facilement par les étudiant(e)s car elle est souvent la première expérience de confrontation à la mort à travers le sujet anatomique. De plus, la mort n'est pas explicitement au programme de l'enseignement de sciences humaines et sociales de première année (PCEM1). Pour les étudiant(e)s IBODE se destinant à une profession s'exerçant au bloc opératoire, cette expérience peut être aussi source de difficulté malgré une pratique antérieure en soins infirmiers. Il s'agit donc de préciser la nature des difficultés rencontrées par ces deux catégories d'étudiant(e)s en identifiant leurs représentations et leurs appréhensions avant, pendant et après les activités de dissection. L'objectif secondaire est de permettre une comparaison entre deux groupes d'âge différent et l'impact éventuel d'expériences antérieures sur le vécu de la dissection et sur l'expression de son ressenti.

\section{Matériel et méthodes}

Pour interroger les étudiant(e)s des deux populations choisies, la méthode du groupe de discussion focalisé ou focus group nous a paru la plus appropriée. En effet, le focus group est une technique d'entretien de groupe qui explore une question spécifique. Son principe repose sur la notion d'expérience commune vécue par l'ensemble des personnes interviewées. La technique du focus group permet d'évaluer des besoins, des attentes, des satisfactions et de mieux comprendre des opinions, des 
motivations ou des comportements. Elle sert aussi à tester ou à faire émerger de nouvelles idées inattendues par le chercheur ${ }^{[1]}$. Les participants sont donc invités à faire part de leurs réflexions à propos d'un thème donné sur la base de leurs opinions et de leur expérience personnelle et/ou professionnelle, chacun étant encouragé à se situer et à réagir par rapport aux avis des autres. C'est ainsi que nous avons choisi de constituer deux groupes de 12 étudiant(e)s, pour deux principales raisons. La première tient au nombre de personnes nécessaire au bon déroulement d'un focus group, nombre variant de 8 à 12 participants afin d'assurer une dynamique satisfaisante et surtout afin de permettre une variabilité de points de vue et d'obtenir des réponses suffisamment représentatives et significatives de la population choisie ${ }^{[2]}$. La seconde raison est liée au fait que la promotion des étudiant(e)s IBODE est de 12. Les entretiens avec les deux groupes ont été enregistrés et leurs contenus ont été dactylographiés afin de recueillir le verbatim des étudiant(e)s qui a ensuite été analysé à partir d'une analyse de contenu thématique permettant ainsi de constituer des catégories ${ }^{[2]}$. Cette analyse a été conduite isolément par un des auteurs sans validation ultérieure par d'autres chercheurs, par les répondants ou encore par des experts. L'analyse des données a été réalisée sans l'aide de logiciel ad hoc, en étudiant les réponses des étudiants et des IBODE pendant l'entretien et en constituant des catégories. Dans le cadre de cette première étude sur un thème tout à fait spécifique et peu exploré, notre démarche s'est positionnée dans une perspective inspirée de celle de la théorie dite ancrée ou enracinée (grounded theory) c'est-à-dire sans cadre conceptuel préalable mais dans une approche inductive par laquelle l'immersion dans les données empiriques a servi de point de départ $^{[3]}$.

Deux focus groups, un de 12 étudiant(e)s en médecine de PCEM2 et un autre de 12 étudiant(e)s IBODE en fin de cursus, ont été exploités pour identifier les représentations et le vécu des participants au sujet des TP de dissections. La grille d'entretien utilisée a permis d'explorer cinq dimensions identi- fiées à partir d'une réflexion entre un enseignant en anatomie et un en psychologie sur la base de leur vécu pédagogique et des questions auxquelles les uns et les autres ont été amenés à répondre aux étudiants. Les cinq dimensions sont les suivantes : 1) les représentations de la mort, du cadavre; 2) l'appréhension et ses manifestations somatiques, psychologiques ou autres avant la séance de dissection; 3) le vécu et les réactions, pensées, comportements devant des cadavres ou pièces anatomiques pendant la séance au laboratoire d'anatomie, de même que les stratégies d'ajustement mises en place pour faire face aux difficultés rencontrées ; 4) les réactions tant immédiates que différées en post-laboratoire et le fait que cette expérience de dissection pourrait avoir une influence sur l'orientation de la carrière des étudiant(e)s de PCEM2 ; 5) les propositions pour améliorer la préparation des TP de dissections.

\section{Résultats}

Ils sont présentés en lien avec chacune des questions explorées au cours des entretiens à partir du guide, en distinguant respectivement les contributions de chacune des deux populations.

\section{Question $n^{\circ} 1$ : représentations du mort} et du cadavre.

Les étudiant(e)s IBODE dont l'âge moyen est de 30-35 ans font une distinction radicale entre un cadavre et un mort. Un cadavre se caractérise par son aspect physique : "c'est un corps froid, raide, dépersonnalisé, c'est un objet avec des organes, qui est mis dans le formol pour la conservation ». Quant au mort, il renvoie davantage à un sujet, à une personne, à une identité ; il y a des affects exprimés autour de la personne morte, avec parfois une dimension spirituelle : «le mort on l'accompagne». Une référence est faite aux rituels qui accompagnent la personne morte. La distinction porte sur le contexte qui va déterminer si on parle de mort ou de cadavre : "professionnellement on parle de cadavre, si c'est personnel c'est un mort. » 
Un étudiant IBODE évoque sa réflexion autour de sa propre mort : «je suis blindé, j'accepte ma propre mort. »

La crainte de tous est la perte du respect concernant le corps à disséquer au laboratoire d'anatomie. La perte de l'aspect sacré de la mort et du mort qui est observée lors des cérémonies est également évoquée. Le moyen de faire face est de faire abstraction de la vie, d'être dans une dimension technique du travail, ce qui permet d'accepter qu'il va y avoir un «délabrement sans réparation possible ».

Suite à cette distinction entre le cadavre et le mort, la question de la connaissance sur la donation du corps est soulevée. Seuls deux étudiant(e)s sur 12 ont des informations sur la démarche du don de corps.

Pour les étudiant(e)s de PCEM2 dont la moyenne d'âge est de 20 ans, la référence au mort est associée exclusivement à leurs expériences personnelles. La moitié des étudiant(e)s au sein du groupe, font état de leurs proches décédés (parents, grands-parents, amis), qu'ils ont tantôt accepté de voir mort ou tantôt refusé, afin d'en garder une image positive. Les dimensions émotionnelles et affectives sont présentes. Quant au cadavre, c'est un sujet anatomique qu'ils se représentent à partir des séries télévisées où il est question de plus en plus de médecine légale. La fiction vient pallier temporairement la réalité.

Aucun des étudiant(e)s n'a connaissance de la démarche de la donation du corps.

\section{Question $n^{\circ} 2$ : manifestations d'appréhension avant la séance de dissections.}

Les étudiant(e)s IBODE ont exprimé peu d'appréhension. La curiosité et l'intérêt primaient sur l'appréhension. "Nous avons déjà été confrontés du fait de notre expérience d'infirmiers dans différents services aux patients en fin de vie et à des personnes mortes, à des cadavres... » ou «Je suis blindée, j'ai eu une réflexion personnelle et j'accepte ma propre mort $»$. Leurs pensées étaient donc davantage centrées sur le laboratoire, son architecture, l'ambiance et sur ce qu'aller apporter ce TP d'un point de vue de l'apprentissage.

Les étudiant(e)s de PCEM2 disent ne pas avoir eu le temps d'appréhender car ils étaient prévenus tardivement de la séance. Ils disent avoir été surtout curieux et intéressés. «Il me tardait de constater si j'allais pouvoir mettre en application les apprentissages théoriques du PCEMI ». Certains se sont d'ailleurs inquiétés de leurs connaissances insuffisantes. Ils disent avoir été davantage centrés sur les aspects matériels (blouses, kit de dissections...) et pédagogiques (évaluation) que sur leurs réactions. Ils ajoutent que ce sont leurs parents qui étaient davantage inquiets qu'eux-mêmes. Ils considèrent ces séances comme un passage obligé en estimant que, s'ils portent un intérêt à la médecine, ils doivent aussi s'intéresser à l' anatomie «Il faut passer par là, c'est obligatoire et c'est là qu'on voit si on est fait pour ça ou pas ».

Question $n^{\circ} 3$ : réactions pendant la séance de dissection.

Les étudiant(e)s IBODE disent avoir été surpris, parfois choqués, devant des corps mutilés, des fragments de corps, des troncs ou des hémi-faces. Même si la plupart ont une expérience, ils ne s'attendaient pas à être confrontés à cela. "C'était quand même choquant, je ne pensais pas que ce serait comme cela... aussi dur ». Ils disent avoir dû se concentrer professionnellement et se rappeler qu'il s'agissait d'un cours d'anatomie. De même qu'ils rapportent avoir fait «corps avec leurs collègues », ils se sont groupés pour se protéger en échangeant paroles, regards, rires... L'odeur a également été difficile à supporter. Ils insistent beaucoup sur la protection du groupe, sur le soutien et le réconfort qu'ils se sont apportés mutuellement. «En fait nous étions proches les uns des autres, cela nous a aidés».

Les étudiant(e)s de PCEM2 se sont d'abord focalisés sur l'ambiance du laboratoire, les néons, les odeurs, les bocaux, tous les éléments donnant un aspect «glauque» au laboratoire, en se demandant si cela était entretenu. La première difficulté a été celle 
de l'incision, le premier contact avec le cadavre et la peur de mal faire et de faire mal. «Le premier coup de lame a été difficile, je n'arrivais pas à enfoncer le scalpel». Pour certains, leurs pensées ont été tournées vers la personne qui a fait don de son corps. Ils ont ressenti la nécessité de respecter ce corp, ce qui a eu pour effet d'inhiber temporairement les gestes techniques à réaliser. Il a ensuite été parfois difficile de se débarrasser des lambeaux de peau et de chair. "À la fin de la séance, il fallait couper la chair en lambeau et la mettre à la poubelle... c'était bizarre ». Pour d'autres, une hyper concentration a permis de s'adapter rapidement et de réaliser les gestes demandé, avec la pensée de ne pas banaliser ces gestes. Les étudiant(e)s se sont d'ailleurs interrogés sur le fait que les techniciens, pour qui c'est le quotidien, puissent être dans la banalisation. Tous disent avoir eu des rires, avoir dit des «bêtises », avoir pensé sur le moment qu'ils disséquaient une «chose» tout en ayant conscience a posteriori que cela était une forme de protection que de ne pas penser à la personne. Certains étudiant(e)s ont remercié la «personne » qui a fait don de son corps et qui leur a ainsi permis d'apprendre l'anatomie. «Moi j'ai dit merci intérieurement à la personne ». "Moi je lui ai donné un nom (rires) c'était marrant ».

Question $n^{\circ} 4$ : réactions après la séance de dissections.

Les étudiant(e)s IBODE rapportent avoir fait des associations entre ce qu'ils ont vu au laboratoire et avec soit des aliments, soit des formes et des couleurs bizarres provoquant parfois des écœurements alimentaires. Pour certains, des images sont revenues pendant un certain temps, d'autres ont fait des rêves. Ceux qui avaient pris des photos lors des séances disent les avoir effacées car les planches anatomiques étaient de meilleure qualité et surtout parce que «cela n'était pas possible». Tous disent que cette expérience n'a pas pu être partagée avec les proches : «ce n'est pas la réalité, c'est un monde à part ». Certains ont pu échanger entre eux, d'autres sont restés sans en parler. Beaucoup se sont interro- gés sur le respect à avoir envers les personnes faisant don de leur corps.

Pour les étudiant(e)s de PCEM2 il est à noter que les réactions ont été mitigées et souvent ambivalentes. Certains sont restés avec un sentiment de malaise et ont fait des rêves au cours desquels ils voyaient des corps découpés, parfois c'est un ressenti qualifié de «bizarre » qui a été exprimé sans plus de précision. Pour d'autres c'est davantage un sentiment de fierté :- «j'ai réussi! »-, le défi ayant été relevé en restant concentré sur l'aspect technique. Il ne fallait pas penser et ainsi se protéger d'un ressenti qui aurait pu parasiter les gestes à réaliser. D'autres encore ont pu se culpabiliser de leur ressenti du moment: "J'ai honte de le dire mais pendant la dissection j'ai eu faim et bien sûr après quand j'y ai repensé j'ai culpabilisé ». Quant à l'influence de ces TP sur leur orientation, ceux qui avaient déjà fait le choix de la chirurgie ont été enthousiastes et ces séances ont pu confirmer leur envie mais aussi et surtout leur capacité à être confronté «au pire». Quant aux autres, ils pensent que cette étape était nécessaire mais qu'elle n'influence en rien leur choix de carrière.

\section{Question $n^{\circ} 5$ : propositions pour préparer} la séance de dissections.

Les étudiant(e)s IBODE expriment qu'il serait souhaitable d'avoir une première séance au cours de laquelle seraient abordées des questions comme celles du don du corps et où des informations pratiques seraient données sur le déroulement de la séance. Concernant les TP de dissections, les étudiant(e)s souhaiteraient commencer à travailler sur un corps entier et non sur des fragments. Une séance de $d e$ briefing après le premier et le dernier TP permettrait d'exprimer son ressenti et d'être rassuré quant au vécu de chacun : "ce serait comme un groupe de parole qui nous ferait du bien, je pense ».

Pour les étudiant(e)s de PCEM2, une première réunion d'information serait souhaitable afin de répondre à de nombreuses questions pratiques. Les questions plus fondamentales comme celles du don 
du corps et de la conservation des corps pourraient être également développées. Les étudiant(e)s demandent aussi des informations quant au déroulement de la séance, au matériel nécessaire pour réaliser les dissections et aux aspects pédagogiques : gestes techniques à réaliser et évaluation. Certains proposent une vidéo ou seraient montrées des dissections; d'autres ne le souhaitent pas et préfèrent «avoir la surprise ». Une petite minorité pense que cela accentuerait l'appréhension. Pour terminer, une séance en post TP pourrait être proposée à ceux qui le souhaitent pour échanger sur leur ressenti et les rassurer : "Est-ce que c'est bien ou mal, normal ou pas d'avoir pensé ou ressenti cela?».

\section{Discussion}

La question des représentations de la mort et du corps mort nous paraissait essentielle à explorer auprès des étudiant(e)s IBODE et de PCEM2 car nous savons que les représentations sous-tendent et participent à nos ressentis, nos réactions et nos comportements. Depuis que les TP de dissections sur du matériel humain existent, il a toujours été observé et fait mention d'étudiant(e)s qui pouvaient être en difficulté et exprimer une certaine anxiété ${ }^{[4-7]}$. Notre étude présente certaines limites au regard des critères de rigueur exigibles dans les approches qualitatives. Ainsi, le critère de crédibilité des résultats aurait pu être mieux satisfait par une triangulation des sources de données, ainsi que grâce à une validation par les répondants des interprétations faites à partir des verbatims. Ces limites étant admises, nos résultats fournissent des informations intéressantes sur de nombreux points. En effet, les étudiant(e)s interrogés, que ce soient les IBODE ou les PCEM2, nous renseignent sur le fait qu'il y a une distinction entre les représentations de la mort et celles du cadavre. Les représentations de la mort sont issues, pour tous, de leurs expériences personnelles. Les représentations du cadavre sont issues pour les IBODE de leur expérience professionnelle et pour les étudiant(e)s de PCEM2 essentiellement de leurs repré- sentations élaborées à partir de fictions (livres, films, jeux vidéos...). Mais pour tous, les TP de dissections permettent de travailler sur un cadavre (corps biologique, anatomique, « corps objet») et non sur un mort (rattaché à la notion de personne).

Si quelques études réalisées auprès de différentes populations d'étudiant(e)s destinées à participer à des TP de dissections sur du matériel humain ont mis en exergue une certaine appréhension de l'exercice ${ }^{[8]}$, les étudiant(e)s interrogés dans notre étude comparative ont exprimé peu d'appréhension. Ces séances obligatoires entrent dans le cadre de leur formation ${ }^{[9]}$ et doivent leur apporter un bénéfice sur le plan de l'apprentissage ${ }^{[10]}$. Nous pouvons penser qu'il y a une intellectualisation qui se fait d'emblée, constituant certainement une défense face à un exercice quelque peu délicat.

Quant aux réactions durant les séances, si un léger malaise est ressenti lors de l'entrée dans le laboratoire et au moment du premier contact avec le cadavre, les étudiant(e)s se concentrent rapidement sur leur tâche à accomplir, sur les gestes à réaliser. Une défense caractérisée par une hyperconcentration permet donc aux étudiant(e)s de se protéger d'une dimension émotionnelle qui pourrait être déstabilisante. De la même manière, le soutien du groupe, qui se manifeste par des échanges de regards, de sourires, de plaisanteries, est vécu comme très important et permet ainsi, grâce à la solidarité, de faire face à une situation des plus spécifiques.

Concernant le vécu «après-coup », si parfois pour certains étudiant(e)s un sentiment de culpabilité émerge du fait d'avoir disséqué une «personne » et non un «cadavre», pour d'autres c'est un sentiment de fierté d'avoir été capable de relever le « défi » et d'être conforté dans leur choix professionnel. Le rite de passage a été réussi et leur appartenance à une communauté est validée. Enfin, tous font état de respect éprouvé face au donateur ${ }^{[11-13]}$ et éprouvent le besoin de recevoir des informations préalables sur le fonctionnement du laboratoire, ce qu'ils vont y trouver, leur rôle, le don de corps ; cette demande s'inscrit dans un intérêt pédagogique 
évident tant pour les enseignants ${ }^{[14]}$ que pour les étudiants ${ }^{[15]}$.

Les résultats de cette étude soulèvent une question plus générale qui est celle du maintien des TP de dissection. En effet, alors que dans de nombreuses facultés de par le monde les séances de dissections disparaissent progressivement, il est légitime de réfléchir à une redéfinition des objectifs pédagogiques assignés à ces séances : doivent-ils être restreints à des apprentissages dans le champ de l'anatomie ou doivent-ils être reconsidérés davantage dans une perspective anthropologique et philosophique qui, au-delà du rôle de rite initiatique que ces activités assument, permettrait d'approfondir diverses notions telles que les concepts de personne ou de dignité, le statut du corps en médecine, etc.

\section{Conclusion}

L'apprentissage de l'anatomie à partir de séances de dissections sur du matériel humain est pour tous les étudiant(e)s de médecine et de bloc opératoire une expérience mettant à l'épreuve leurs représentations et suscitant une certaine forme d'appréhension. Et si très rapidement les manifestations d'appréhension sont remplacées par un processus d'hyperconcentration permettant à chacun, dans une dynamique protectrice, de réaliser des gestes techniques, il n'en demeure pas moins qu'une réflexion émerge sur la dissection et sur le ressenti éprouvé. Tous les étudiant(e)s ont conscience de la chance qui leur est offerte de pouvoir bénéficier de telles séances ${ }^{[15,16]}$, soulignant l'apport évident du matériel humain au plan anatomique, chirurgical, technique dans le cadre de leur formation professionnalisante. C'est pourquoi ils sont unanimes à dire qu'il faut continuer de bénéficier de cet apprentissage qui pourrait cependant être préparé par un premier temps essentiellement informatif avant les séances et par un temps d'expression libre, encadré, à l'issue des séances, afin de pouvoir échanger et comparer un vécu, et ce afin de se rassurer. C'est ainsi qu'une séance d'information pédagogique ainsi qu'un groupe de debriefing post-TP pour les volontaires ont été mis en place auprès des étudiant(e)s de PCEM2 et IBODE dès la rentrée académique 2007-2008. L'objectif est donc d'une part de pallier un manque d'information qui pourrait être anxiogène et, d'autre part, de préparer les étudiant(e)s à une épreuve peu banale et ainsi tenter de prévenir certaines angoisses et répercussions psychologiques.

\section{Remerciements}

Nous remercions les étudiants de PCEM2 de la faculté de médecine de Nantes et les étudiant(e)s infirmier(e)s de bloc opératoire du CHU de Nantes de l'année universitaire 2006-2007.

\section{Contributions}

Angélique Bonnaud-Antignac et Olivier Armstrong ont conçu le protocole d'étude, mis en œuvre les méthodes de recueil de données et interprété les résultats. Ils ont rédigé conjointement les versions successives du manuscrit. Antoine Hamel, Jean-Michel Rogez, Dominique Guilloton, Yves Maugars et Jacques-Henri Barrier ont été associés à la réflexion initiale concernant la problématique de recherche et leurs avis ont été sollicités à propos des versions initiale et finale du manuscrit.

\section{Références}

1. Moreau A. S'approprier la méthode du focus group. Revue du praticien de médecine générale 2004;645:382-384.

2. Duschene S. HF. L'enquête et ses méthodes : l'entretien collectif. Paris : Nathan, 2004.

3. Guillemette F. L'approche de la Grounded Theory ; pour innover ? Recherches Qualitatives 2006;26:32-50.

4. Horne DJ de L, Tiller JWG, Eizenberg N, Tashevska M, Biddle N. Reactions of first year medical students to their initial encounter with a cadaver in the dissecting room. Acad Med 1990;65:645-6.

5. Dinsmore CE, Daugherty S, Zeitz HJ. Student responses to the gross anatomy laboratory in a medical curriculum. Clinical Anatomy 2001;14:231-6. 
6. Arráez-Aybar LA, Casado-Morales MI, Castaño-Collado G. Anxiety and dissection of the human cadaver: An unsolvable relationship? Anat Record (Part B: New Anat.) 2004;279B:16-23.

7. Sanner MA. Encountering the dead body: experiences of medical students in their anatomy and pathology training Omega J Death Dying 1997;35:173-91.

8. Dickinson GE, Lancaster CJ, Winfield IC, Reece EF, Colthorpe CA. Detached concern and death anxiety of first-year medical students: before and after the gross anatomy course. Clinical Anatomy 1997;10:201-7.

9. Roland J. Formation des médecins. Les médecins aujourd'hui en France. Actualité et dossiers en santé publiques (ADSP) 2000;32;24-6.

10. Barrier JH, Brazeau-Lamontagne L, Colin R, Quinton A, Llorca G, Ehua FS et le conseil pédagogique de la Conférence Internationale des Doyens des Facultés de Médecine d'Expression Française. La formation au professionnalisme des futurs médecins. Recommandations du Conseil Pédagogique de la CIDMEF. Pédagogie Médicale 2004;5:75-81.

11. La charte de l'éthique des facultés de médecine : un cadre conceptuel et un outil. Pédagogie Médicale 2004;5:197-99.
12. Charte de l'éthique des facultés de médecine. Conférence Internationale des doyens et des facultés de médecine d'expression française (CIDMEF). Pédagogie Médicale 2004;5:211-7.

13. Brazeau-Lamontagne L, Barrier JH et Ehua SF. Application de la charte de l'éthique des facultés de médecine : définition des premières orientations stratégiques. Pédagogie Médicale 2006;7:59-62.

14. Roland J. La pédagogie dans les facultés de médecine françaises. Pédagogie Médicale 2001;2;6-8.

15. Bakinde N, Mooser F, Voigt-Gawatz T, Nkumbe H, Rager G. Enseignement de l'anatomie : ce que les étudiants en pensent. Bull Soc Frib Sc Nat 2005;94:77-87.

16. McGarvey MA, Farrell T, Conroy RM, Kandiah S, Monkhouse WS. Dissection: a positive experience. Clinical Anatomy 2001;14:227-30.

Correspondance et offprints : Angélique Bonnaud-Antignac. Département de sciences humaines et sociales, Faculté de médecine de l'université de Nantes, 1 rue Gaston Veil, 44093 Nantes Cedex, France.

Mailto : angelique.bonnaud@univ-nantes.fr 